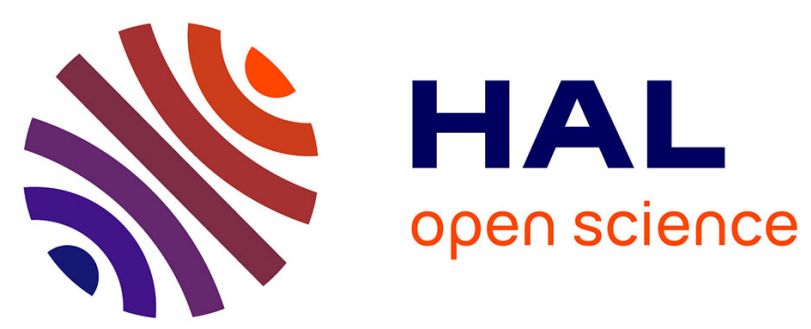

\title{
NONLINEAR OPTICAL EFFECTS AT THE FUNDAMENTAL ABSORPTION-EDGE OF WIDE-GAP II-VI SEMICONDUCTORS
}

F. Henneberger, J. Puls, H. Rossmann, Matthieu Kretzschmar, C. Spiegelberg, A. Schülzgen

\section{- To cite this version:}

F. Henneberger, J. Puls, H. Rossmann, Matthieu Kretzschmar, C. Spiegelberg, et al.. NONLINEAR OPTICAL EFFECTS AT THE FUNDAMENTAL ABSORPTION-EDGE OF WIDEGAP II-VI SEMICONDUCTORS. Journal de Physique Colloques, 1988, 49 (C2), pp.C2-91-C2-96. 10.1051/jphyscol:1988220 . jpa-00227637

\section{HAL Id: jpa-00227637 https://hal.science/jpa-00227637}

Submitted on 1 Jan 1988

HAL is a multi-disciplinary open access archive for the deposit and dissemination of scientific research documents, whether they are published or not. The documents may come from teaching and research institutions in France or abroad, or from public or private research centers.
L'archive ouverte pluridisciplinaire HAL, est destinée au dépôt et à la diffusion de documents scientifiques de niveau recherche, publiés ou non, émanant des établissements d'enseignement et de recherche français ou étrangers, des laboratoires publics ou privés. 
F. HENNEBERGER, J. PULS, H. ROSSMANN, M. KRETZSCHMAR, C. SPIEGELBERG and A. SCHÜLZGEN

Humboldt-Universität zu Berlin, Sektion Physik, Bereich 05 , Halble1teroptik, Invalidenstr. 110, DDR-1040 Berlin, D.R.G.

Abstract: This papers summarizes recent results on nonlinear absorption and refraction, optical switching bistability and digital logics at visible wavelengths using wide-gap semiconducting compounds.

\section{i - INTRODUCTION}

Previous studies $/ 1,2 /$ have shown that very strong and fast optical nonlinearities occur near the fundamental absorption edge of the wide-gap II-VI semiconductors at room temperature. They are related to the disappearance of the exciton resonances due to free carrier screening and make these materials good candidates for optical signal processing applications in the visible spectral range. This paper summarizes some results of more recent studies in this area.

\section{2 - NONLINEAR ABSORPTION AT MW-POWER LEVELS}

Previousty $/ 1,3,4 /$ we have demonstrated saturable absorption of CdS, CdSe and the corresponding mixed crystals in a near steady-state regime both in excite and-probe and single-beam experiments with nanosecond dye laser pulses at intensities of a fow $\mathrm{kW} / \mathrm{cm}^{2}$. Those intensities are gainable by the output of $\mathrm{cw}$ laser sources focused on spots of some $\mu \mathrm{m}$ diameter and, in fact, we have recently succeeded in achieving full steady-state operation of the nonlinearity with the Argon-lon laser. Oy means of an acousto-optical modulator 50 ns pulses were formed. The nonlinear transmission at two laser lines is shown in Fig. 1 . Bleaching of absorption at mW-power levels is clearly seen. This is one of the rare examples where a fast optical nonlinearity of electronic origin can be operated with $\mathrm{cw}$ lasers at room temperature. Since the switching times are clearly pico- and subpicosecond (see below) the switching energy is on the $\mathrm{fJ}$-scale. Using the $\mathrm{Ar}^{+}$laser in a modelocked regime or the ps-YAG laser alloptical logic operations (AND, OR) have been performed in a pulsed mode. Current work concentrates on heat reduction to increase cycle rates.

\section{3 - DEGENERATE FOUR WAVE MOXING AND NONLINEAR REFRACTION}

Amy change of absorption is accompanied by a corresponding change of refraction. A Kramers-Kronig anabysis $12,5 /$ of nonlinear absorption data has ylelded values as large as $6 \times 10^{-19} \mathrm{~cm}^{3}$ for the change of refraction $\delta n$ induced by one electron-hole pair per $\mathrm{cm}^{3}$ in CdS. However, the accuracy of Kramers-Kronig calculations is somewhat questionable, since there can be wak, but very long-ranged non-resonant absorptlon changes. In order to confirm this large nonlineer refraction experimentally, wo have carried out degenerate four- wave mixing experimente. Two beams of equal intensity $I_{0}$ from a dye laser operating at a power-level of some kW and pulse durations of $*$ ins were made to interfere on a $1.0 \mathrm{\mu m}$ CdS platelet under a certaln angle. The perlod of the resulting grating was about $20 \mu \mathrm{m}$, which is claarly larger than the carrier diffusion longth. The intensitios of the incoming baams all as the transmitted $\left(I_{T}\right)$ and the first-order four-wave mixing signals $\left(I_{p a t}\right)$ were detected at the maximums of the pulese by fast Si-photodiodes and a boxcar integrator yialding a time resolution better then ins.

Fig. 2 presents the absolute four-wave mbing efficiencies observed scanning the frequency of the incoming light through the band-edge region. Maximum efficiency occurs somenthat below the groundstate exciton resonance 
and a value of $5 \%$ is found at highest pump levels. For the ratio $I_{p=1} / I_{T}$ we get even values up to $20 \%$ around $2.5 \mathrm{oV}$. The scattering of the data at the low - energy side at highest pump is probably related to Fabry-Perot effects. Here, the refraction changes cause already substantial shifts of the interference fringes of the plateletlike sample. The pump intensity is that high that the nonlinearity is mainly due to bandfilling as evidenced by the
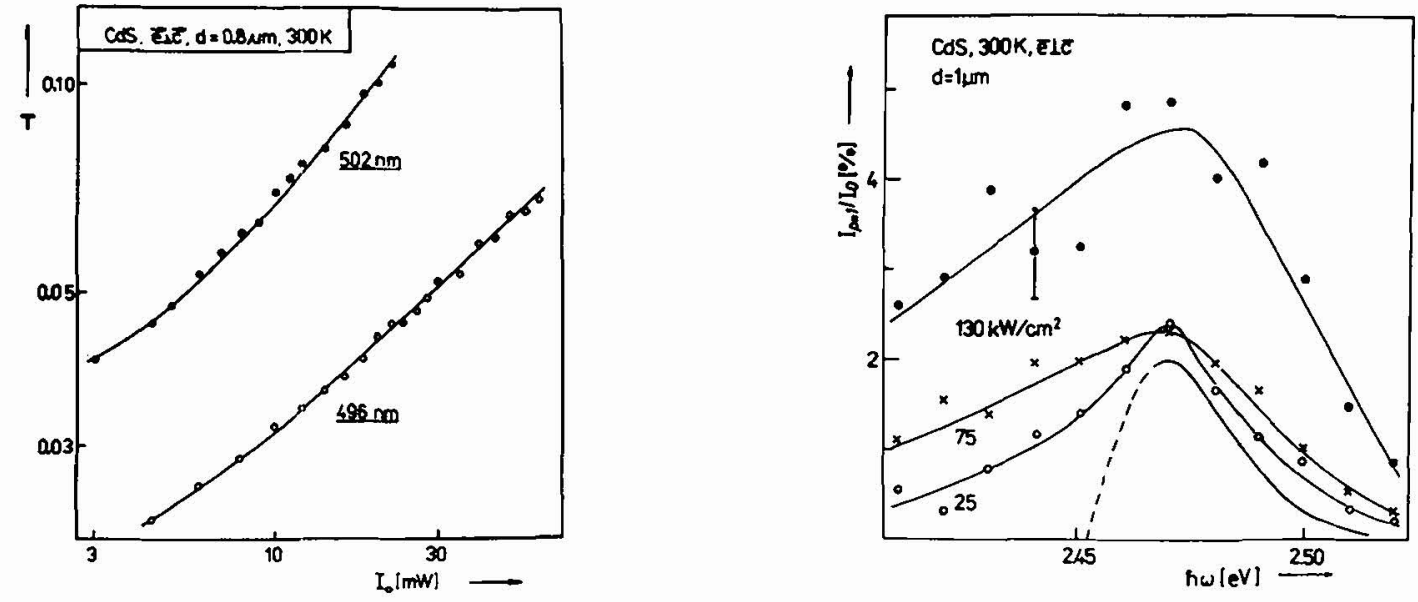

Fig. 1 Transmission of a $0.8 \mu \mathrm{m}$ CdS sample at $\mathrm{T}=300 \mathrm{~K}$ versus input power at two wavelengths of the $\mathrm{Ar}^{+}$laser. Polarization perpendicular to the hexagonal axis.

Fig. 2 First-order degenerate four-wave mixing efficiency versus photon energy of a $1.0 \mu \mathrm{m}$ thick CdS sample at $T=300 \mathrm{~K}$ for three different input intensities. Polarization of all beams perpendicular to the hexagonal axis. Lowest curve: Calculated efficiency of the amplitude grating at $25 \mathrm{~kW} / \mathrm{cm}^{2}$ using the nonlinear absorption of $/ 1.2 /$.

appearance of etimulation effects in the luminescence spectrum. In addition, pump depletion sets in. Therefore, for a detailed anabysis we concentrate on lower pump lovels. As known from earlier experiments /1,2/ there is strong nonlinear abeorption decrease on the high-energy side. Using the respectlve data we have calculated the diffraction from the corresponding amplitude grating and good agreement with the experiment is obtained in this spectral range. On the other hand, only a weak absorption change is observed on the low energy side. Accordingly. the diffraction results here from a phase grating. from which one can determine the change of refraction $\Delta$. $\Delta$ between the dark and bright spots of the interference grating is given by /6/

$$
2 \Delta n=2(\lambda / d)\left[(1-R)^{-2} \exp (\alpha d) I_{p=1} / I_{0}\right] 1 / 2
$$

( $R=0.2$ is the reflection, $d=1 \mu \mathrm{m}$ the sample thickness, and $\alpha$ the Inear absorption measured simultaneousty). in addition, the carrier density at the interference maximums can be estimated from

$$
n_{1}=4 I_{0}(1-R)(\tau / h \omega d)[1-\exp (-\alpha d)] .(2)
$$

( $\tau$ is the carrier Hfe - time of 200 ps /1,2/.) Combtning both equations we can deduce the change of refraction $\delta n=2 \Delta \mathrm{n} / \mathrm{n}_{\mathrm{f}}$ induced by on electron hole pair $\mathrm{m} 1 \mathrm{~cm}^{3}$. The result is shown $\mathrm{m} \mathrm{Fg} .3$ and, in fact, values as large as predicted by the Kramers - Kronig anabysis are found. Surpriaingly, the nonlinear refraction docreases at higher pump. We attribute this to a trensition to a bandfilling nonlinearity, which is not that effoctive than the excitonic one.

Our four-wave mixing studbs demonstrate that CdS exhiblte very large nonlinear rotraction at room temperature. Athough our sample be very thin, efficiencies of some percent have been observed. The nonlinear refraction cosfficiont in ts as large as that of GaAs/GaAlAs multiple quantum wells $/ 7 /$. However, the present data have been obtalned in a quasl steady-etate reakne; in a spectral ranee of substantialy lower lnear absorption 


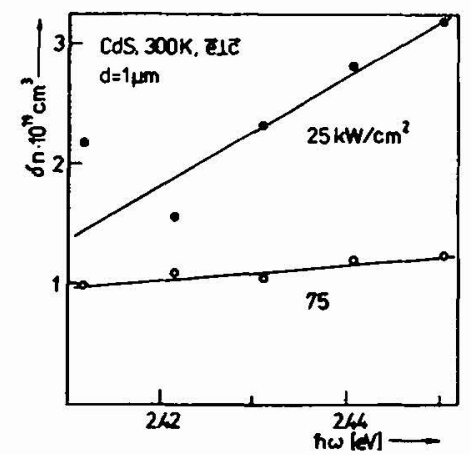

Fig. 3 Change of refraction per electron - hole pair per $\mathrm{cm}^{3}$ at the low - energy side of the exciton groundstate resonance ( $E_{x}=2.475 \mathrm{eV}$ ) calculated from the data in Fig. 2 and (1) and (2).

and for a 100 times shorter recovery time. These large refraction changes make CdS a very good candidate for applications in dispersive optical bistability, phase conjugation, and other reiractive switching techniques at visible wavelengths.

\section{4 - FEMTOSECOND DYNAMICS}

Picosecond excite and-probe measurements $/ 2,8,9 /$ have yielded carrier-recombination controlled recovery of the nonlinearity within 200 ps. Switch-on occurred instantaneously with the pump pulse of 6 ps. Thus, the underlying process is shorter than this time and a study on the fs time-scale is desirable.

Using the amplified output of a CPM laser (') at $618 \mathrm{~nm}$ with a pulse duration of 115 fs we have studied in a excite- and-probe configuration:

(i) Below-gap , exciton resonant excitation of $\mathrm{CdS}_{x} \mathrm{Se}_{1-x}$ matching $x$ property and

(ii) above-gap excitation of pure CdSe.

The pump polarization was chosen parallel to the crystal axis c, so that primary onty B-holes were excited. In accord with earlier experiments on the ns- and ps-time scate /1,2/ we have observed in both situations a pronounced increase of the probe transmission. however. with a different time behaviour. For exciton-resonant

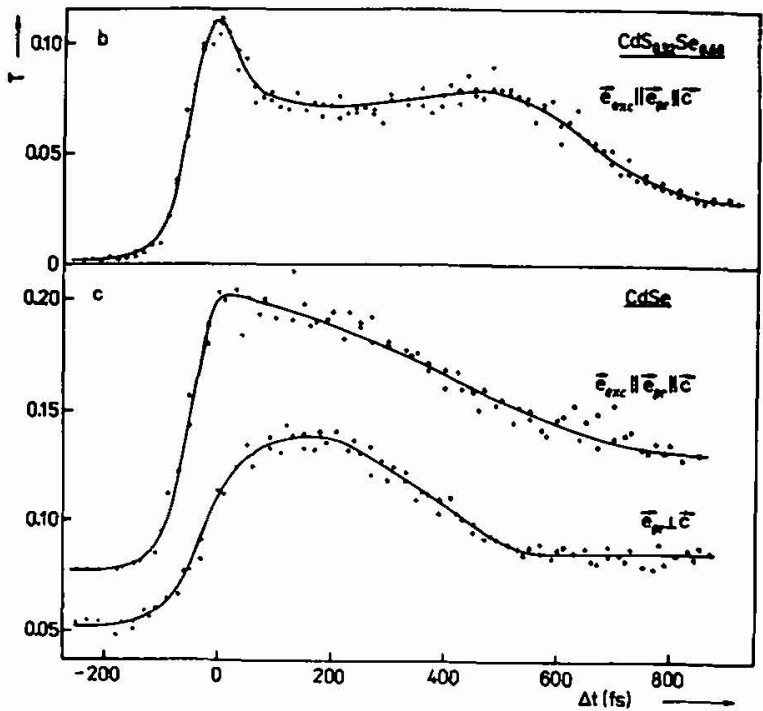

Fig. 4 Probe beam transmission versus pump-probe delay (Pump $1 \mathrm{~mJ} / \mathrm{cm}^{2}$ Probe: $0.01 \mathrm{~mJ} / \mathrm{cm}^{2}$ ).

a) B-exciton resonant excitation of $\mathrm{CdS}_{x}$ Se $_{1-x}$ (thickness $3 \mu \mathrm{m}$ ). A value of $x=0.34$ is used to match the $618 \mathrm{~nm}$ laser output.

b) $250 \mathrm{moV}$ above- gap excitation of CdSe $(0.3 \mu \mathrm{m})$. All data at room temporature.

\footnotetext{
1 The fe-studiea heve been performed in close cooperation with M. Rudolph and co-workers at the FriedrichSchiller-Univeraltät, Jena (GDR).
} 
excitation (Flg, 4a) we find a distinct coherence poak, followed by a plateau and subcequent recovery with a time constent of about $250 \mathrm{fs}$. ( On a commercial fltter of CdS $\mathrm{Se}_{1-x}$ microcryetalltes embedded in glase otudiod for comparison no recovery is seen within 1 ps 1 . To eeparate coherent interaction and that originating from real carriers we have studied the first-order defraction from the transtent grating produced by pump and probe togother. A slight asymmotry of the efficiency ( with respect to probe ahead or behind pump) is found, which we attribute to the exciton dephasing with a characteristic time of about 50 fa. Substracting the coherence part in Fig. La we get a switch-on time for the nonlinearity via carrlers somewhat bolow 100 fs. Plysically, this is the time neceseary for the ionization of excitons by LO-phonon sbeorption resulting in free carriers screening out the exctton resonance. Simultanoously the gap shrinks, so that the actual band edge fo bolow the exchation frequency at larger delays and a complex carrier dynamice sets h leading to a nearly constant probe transmiselon. The very fast recovery results from the decay of the non-thermal carriers at the excitation frequency down to the band bottoms (c.f. Fig. 4b). Note, that at room temperature there le no essential difference between the Inear lovele of the below-gap exciton and above-gap continuum absorption.

For 250 meV above-gap excltation of CdSe (Fig. 4b) no clear coherence peak is seen , since wo produce directhy carriers with very rapid dephasing: the first-order efficiency follows instantaneously the pump pulse in this case. We observe essentlally the state-flling dynamics of the carriers studied at low temperature in $/ 10 /$. However, we find different results for probe polarization parallel and perpendicular to $c$. In the first case ( upper curve in Fig. 4b) the absorption blocking due to B-holes is probed and, accordingly, no delay of the respective tranemision increase is seen. But, using probe polarization perpendicular c, wo monizor the blocking due to A- holes, which, in fact, shows up delayed to that of 8-holes. Thus, we have directly observed the 8- to A-hole conversion in CdSe which takes about 80 fs.

The data in Flg. 4 directly demonstrate that the wide-gap II- VI's can be used for optical switching and logic operations in the subpicosecond range. High contrast, fs-recovery, and room temperature distinguish them from other materials.

\section{5 - OPTICAL BISTABILITY}

The nonlinear absorption and refraction discuseed above allows to achieve Fabry-Perot bistability on reflection coated samples. Operating at the exciton resonace $(h \omega=2.475 \mathrm{eV}$ at $e \perp c)$, where the absorption bleaching is maximum, we have demonstrated absorptive FP bistablity in the $\mathrm{kW} / \mathrm{cm}^{2}$ intensity range on CdS at room temperature $/ 1,2 /$.

The data in Chapter 2 suggest dispersive FP bistability far away from the exciton resonance. We have studied an etalon made from a dielectrically coated CdS sample the transmission peak of which is 70 meV (!) below the exciton resonance (see insert Fig. $5 \mathrm{a}$ ). Since the nonilinear refraction is negative in this regton, the laser was tuned on the high energy side. so that the peak runs to the laser frequency with rising intensity. The shapes

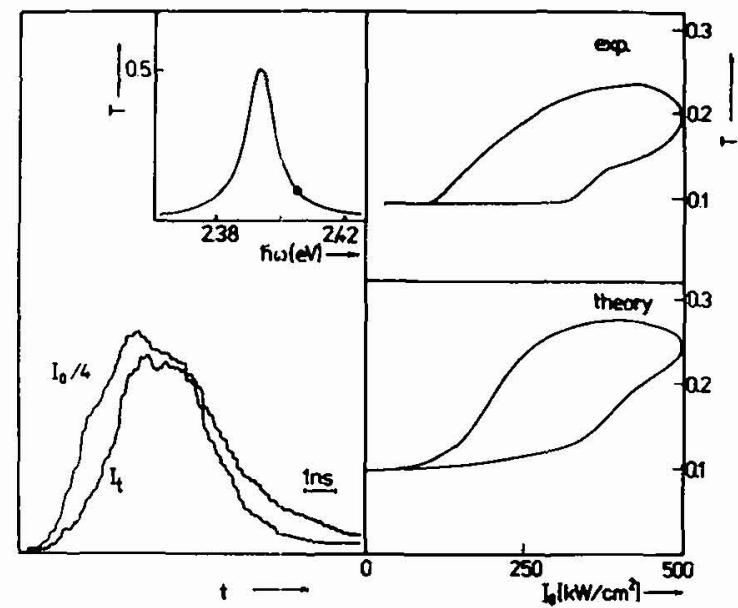

Fig. 5 Dispersive Fabry-Perot bistability on dielectrically coated $C d S(d=1.1 \mathrm{um})$ at room temperature. Explanations see text 
of the Incident and trasemitted pules is shown in the lower loft and the corresponding transmission versus input intensity loop in the upper right part of Fig. 5, respectively. Hysteresis occurs only above some certain peak Intensity. while nonlinearity is already observed at smaller inputs. Thus, the loop in Fig. 5 is not a simple transient one. However, no claar switching is seen. This is due to transverse effects, because the spot $(\approx 35 \mu \mathrm{m})$ is much larger then the cerrier diffusion length. This is confirmed by a respective calculation/11/treating the full spatial ( in both directions parallel and perpendiculer to the beam ) and time problem for the carrier density and ight intensity. The result is presented in the lower right part of Fig. 5 and good agreement with the experimental loop is seen. The only free parameter is the carrier diffusion length for which a value of 1 um is used being in reasonable accord with literature data $/ 12 /$. The Intenslties needed to get bistablity are more than 10 times larger as compared to the absorptive case /1,2/. However, since only $8 \%$ of the input intensity are absorbed, the energy consumption is equal for both kinds of bistablitios. Similar bistable behaviour at thicker samples and higher intentensitios has been reported in $/ 13 /$.

The nonlinear Fabry-Perot cavity is only one way to achive bistablity and for both fundamental and practical reasons, there is an extensive search for now concepts, which do not require external mirrors. One of the most easy ways to achieve mirrorlose bletabilty is increasing absorption (see e.g. $/ 14$ / ). However, from a practical point of vlow the high absorption necessarily imolved is of disadvantage. Soveral papers $($ for references see /44/ or /15/) have proposed and partly demonstrated dispersive cavityless bistability, but none of them is substantially superior to the Fabry-Perot cavity. A virtually simple concept would be nonlinear reflection at the boundary between different media provided positive feedback can be put into action. That concept has been
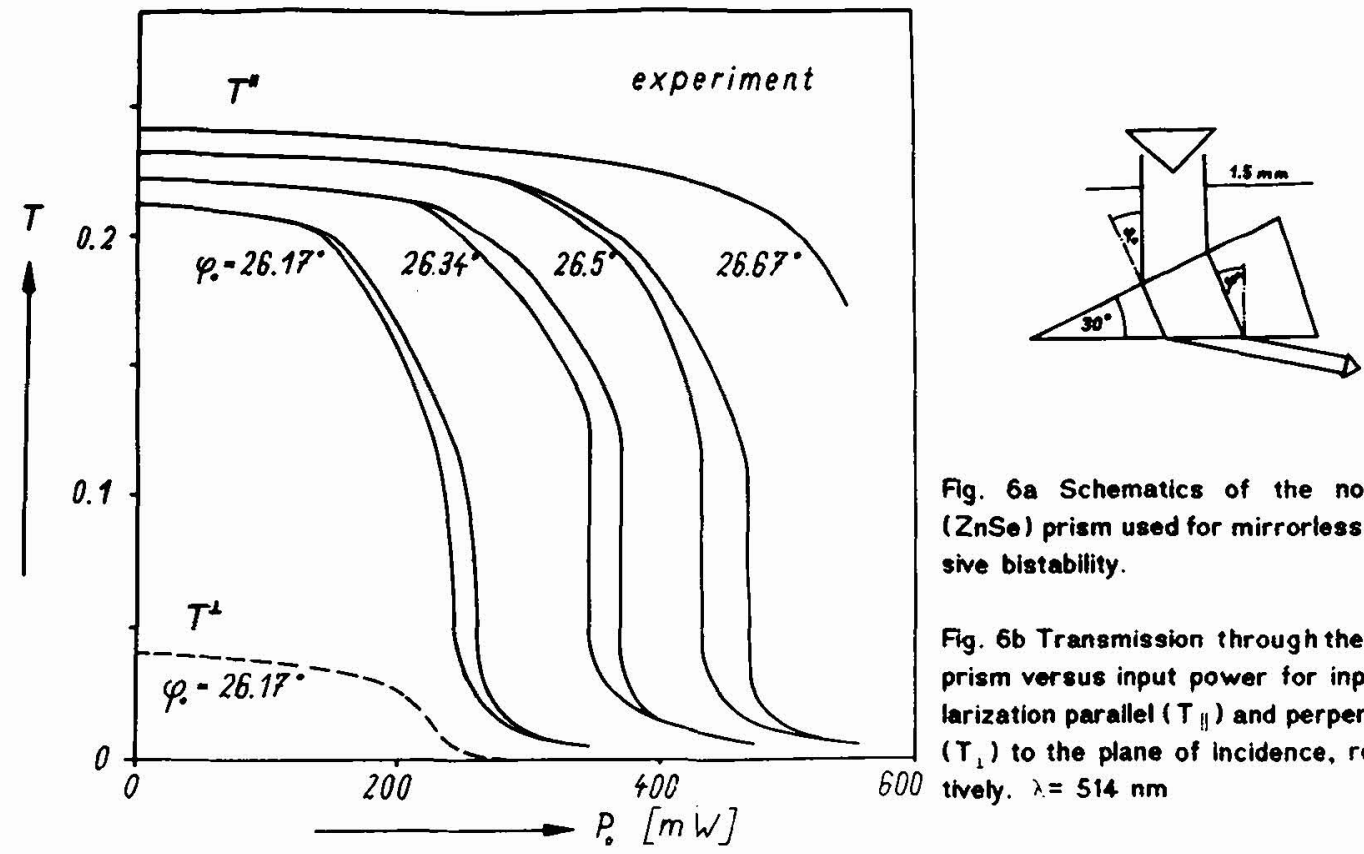

Fig. 6a Schematics of the nonlinear ( $\mathrm{ZnSe}$ ) prism used for mirrorless disper sive bistability.

Fig. 6b Transmission through the above prism versus input power for input polarization parallel $\left(T_{\|}\right)$and perpendiculat $\left(T_{1}\right)$ to the plane of incidence, respectively. $\lambda=514 \mathrm{~nm}$

firstly used in $/ 16 /$, where the specific situation of total-reflection incidence on an optically thin medium with nonlinear refraction has been considered. We have recently proposed and demonstrated $/ 15 /$ a new kind of mirrorless dispersive bistability also based upon nonlinear reflection, but distinctly different to that of $/ 16 /$. The general schematics of that bistability is shown Fig.6a. At first sight it looks similar to that used in $/ 16 /$, but in these studies the nonlinear medium is placed under the output face of the prism. The latter is only needed to achieve total reflection at the nonlinear interface at low intensities. In contrast, we consider the situation, where the prism itself presents the nonilinear medium merely surrounded by air. A second point cruclaily for the bistability presented here is that we use an input beam polarized in the plane of incidence. Then. the reflection at the prism output face changes drastically (ideally between Zero and unity) when the angle of incidence is tuned from the 
Breweter angle $\varphi_{\mathrm{Q}}$ into that for totel refiection $\varphi_{T}$. For the prism we use a modium with pocitive nonilnear refractior $n=n_{1}+n_{2}$ l. Accordingly, $\varphi_{B}$ and $\varphi_{T}$ move to lower angles with riaing light intensity 1 . The input bean be now implinged on the priam in a way ylelding $\varphi_{Q} * \varphi^{*}<\varphi_{T}$ at low intenaitles, $\varphi^{\text {* }} k$ the angle of incidence on the priam output face. Thet $b$, the ight incident on the output face of the prism almost fully transmitted and the nonlimarity is onty driven by the input beam $\left(I \approx I_{0}\right)$. Increasing of the input intensity increases the actual $n$. whereby $\varphi_{B}$ shifts offwards but $\varphi_{T}$ towards $\varphi^{*}$ and the reflection increases. The reflected Hght increases in turn the refraction index of the prism, so that $\varphi_{T}$ approaches more closeby $\varphi$ *resulting in a further increase of the reflection and so on. If the reflection increase is steep enough this feodback becomes regenerathe bading to runaway and switching to total refiection. Now the nonlinearity is pumped by the incoming and reflected bean $\left(1 \approx I_{0}+I_{R} \approx 21_{0}\right)$. When the input intensity is subsequently reduced the reflected signal hetps the prism to maintain total refiection resulting in opticel hysteresis. For the experimental demonstration of this bistablity wo heve used anSe prism and the $514 \mathrm{~nm}$ line of the Ar laser $\left(\varphi_{\mathrm{B}}=19.78^{\circ}\right)$. The nonlinearity is of thermal origin. A detailed theoretical analyais $/ 15 /$ shows that blstabilty is expected for input angles $\varphi_{0}$ lerger than $26^{\circ}$ for the priam apex angle of $30^{\circ}$ used. Indend, we find in the experiment clear bysteresis just in that range of input angles (Fig. $6 \mathrm{~b}$ ). To check, wether this is not simply increasing absorption bistability we have tuned the polarization of the input beam in the direction perpendicular to the plane of incidence and, as expected, hysteresis disappeared. Bistable switching at angles of incidence larger than $27^{\circ}$ could not be demonstrated because of the available maximum power of the laser. Note, however, that the intensity on the sample is as low as $8 \mathrm{~W} / \mathrm{cm}^{2}$ since we operate without focusing. Hysteresis occurs also in the output angle. This feature is different to the Fabry-Perot cavity and of practical Importance, since it can be used in several ways to direct or adress beams. In addition, the advantage of this bistability in comparison to the previously studied bistability at total reflection $/ 16 /$ is that wo operate in a regime, where the input light penetrates fully into the nonliniear medium and, thus, pumps much more offectively.

The cavityless dispersive bistability demonstrated above shows another useful property of the wide-gap II-VI semiconductors. These materials make available thermo-optic nonlinearities at the wavelengts of the Ar ion laser. Therefore, they are well sulted to study in en easy way the behaviour of bistable systems or to demonstrate even now types of optical bistabilities.

\section{REFERENCES}

1/ Henneberger, F., Puls, J., and Spiegelberg, Ch., Optical Bistability III, Ed. Gibbs, H.M., Mandel, P., Pheyghambarian, N.and Smith, S.D., Springer-Veriag 1985 (p. 156).

12/ Henneberger. F.. Puls. J., Rossmann. H., Spiegelberg. Ch., Kretzschmar, M., and Haddad, 1.. Proc 6.th General Conf. Condensed Matter Division Europian Phys.Soc., Physica Scripta T13 (1986) 195.

/3/ Puls, J. and Henneberger, F., phys. stat. sol. (b) 121 (1984) K187.

/4/ Henneberger. F., Puls, J., and Rossmann, H., J. Lum. 30 (1985) 204.

15/ Henneberger, F., Woggon, U., Puls, J., and Splegelberg, Ch., Appl. Phys. B 45 (1988) in press.

$16 /$ Eichler, H.J., Festkörperprobleme 18 (1978) 241.

$17 /$ Miller, D.A.B., Chemla, D.. Eilenberger, D.J., Smith, P.W., Gossard, A.C. and Wiogmann. W., Appl.Phys. Letters 42 (1983) 295.

18/ Puls, J., Fink, F., and Henneberger, F., Proc. Internat. Symp. Ultrafast Phenomena Spectroscopy, Minsk 1983, p. 339.

19/ Puls, J., Henneberger, F., Fink, F., and Jipner, I., Proc. Internat. Symp. Uitrafast Phenomena Spectroscopy. Reinhardsbrunn (GDR).1985 p. 339.

$110 /$ Peyghambarian, N., and Koch, S.W. , Rev. Phys. Appl. 22 (1987).

$111 /$ Kretzschmar, M..thesis, Humbotdt-Universität, Berlin 1988.

/12/ Saito, H. and Gobel,E.O., Phys.Rev. B 318 (1985) 2360.

Majumder,F.A., Swoboda, H.E. ,Kempf, K., and Klingshirn, C., Phys. Rev. B32 (1985)2407.

113/Wegener, M., Klingshirn, C., Koch, S.W. , and Baryaj,L. . Semicond. Sci. Technol. 1 (1986)366.

$/ 14 /$ Henneberger, F. , phys. stat. sol. (b) 137 (1986) 371.

115/ Henneberger. F., Rossmann. H., and Schuizgen. A., phys. stat. sol. (b) 145 (1988) K83.

116/ Smith. P.W. Tomlinson, W.J., Maloney. P.J.,and Hermann. J.P. IEEE J. Quantum Electronics 17 (1981) 381. 